\title{
The Features of Adrenal Tuberculosis by Contrast-Enhanced
}

\section{Ultrasound}

\author{
Gaoyi Yang, 1," Wenzhi Zhang, ${ }^{1}$ and He Ning ${ }^{1}$ \\ ${ }^{1}$ Department of Ultrasonography, Hangzhou Red Cross Hospital, Hangzhou, China \\ "Corresponding author: Gaoyi Yang, MD, Department of Ultrasonography Hangzhou Red Cross Hospital, No 208, Huangcheng East Road, Hangzhou, China, 310003, E-mail: \\ yanggaoyi8@163.com
}

Received 2016 November 28; Revised 2016 December 22; Accepted 2017 February 15.

Keywords: Adrenal Tuberculosis, Contrast-Enhanced Ultrasound, Sonography

\section{Dear Editor,}

Extrapulmonary tuberculosis has been increasing in the past decades and accounts for approximately $20 \%$ of the newly diagnosed tuberculosis cases (1). The most common tuberculosis of the endocrine system is adrenal tuberculosis, which contributes to $20 \%-30 \%$ cases of Addison's disease, a chronic endocrine system disorder (2). Early diagnosis of adrenal tuberculosis is difficult due to the invisible symptoms at early stages of the disease (3). In the present study, we aimed to evaluate the application of contrast-enhanced ultrasound (CEUS) in the diagnosis of adrenal tuberculosis in Chinese patients.

From January 2010 to July 2015, eighteen patients (12 men and 6 women) confirmed as adrenal tuberculosis with histopathology of surgery specimen were recruited. The study was approved by the Institutional Ethic Review board. We used a Philips IU22® Ultrasound machine (Philips, Amsterdam, the Netherlands) for the diagnosis, with a C5-1 probe and frequency setting as 1.0 - 5.0 MHz. We defined them as hypoechoic lesions if the echo of the adrenal lesion was lower than that of the surrounding glandular tissue or normal renal cortex, mixed-echoic lesions if liquid or calcification was observed, and hyperechoic lesions if there were various types of calcifications in the lesions. Punctate calcification was confirmed if the diameter of the calcified lesion was equal or less than $0.2 \mathrm{~cm}$. A diameter more than $0.2 \mathrm{~cm}$ was defined as coarse calcification.

Low mechanical index and pulse inversion harmonic imaging were used for the CEUS examination, and SonoVue (Bracco Diagnostics Inc, Italy) was used as the contrast agent. The CEUS examination was categorized into three stages: early arterial phase ( 0 to 20 seconds after the injection), arterial phase (21 to 40 seconds after the injection), and parenchymal phase (41 to 300 seconds after the injection) (4-6). We then categorized lesions into homogeneous and heterogeneous enhancement based on the enhancement patterns, and into hypo-enhancement, hyper-enhancement and iso-enhancement based on the enhanced intensity.

The mean age of the patients was $56.7(\mathrm{SD}=12.8)$. Results of the conventional sonography suggested that 13 patients had the lesions on both sides and five patients only on one side. The mean size of the lesions was $4.5 \mathrm{~cm}$ (SD $=0.8 \mathrm{~cm}$ ) (a total of 31 lesions). We found that 17 of the 31 lesions (54.8\%) appeared as mixed-echoic lesions (Figure $1 \mathrm{~A}$ and Figure $2 \mathrm{~A}$ ), and the other 14 cases (45.2\%) were hypoechoic lesions (Figure 3A). Punctate, lamellar or coarse calcifications were observed among 19 lesions (61.3\%), and different types of calcifications may co-exist within a single lesion. Color Doppler flow image (CDFI) did not show any signal in 29 (93.5\%) of the lesions.

After the injection of contrast agent (for CEUS), 26 of the 31 lesions (83.9\%) showed enhancement in the early arterial phase, with an average contrast agent arrival time of $10.7 \mathrm{~s}(\mathrm{SD}=2.6 \mathrm{~s})$. Among the 26 lesions, 14 started to washout in the arterial phase, and 12 started to washout in the parenchymal phase. The average washout time was $38 \mathrm{~s}(\mathrm{SD}=6 \mathrm{~s})$. Five of the 31 lesions $(16.1 \%)$ showed nonenhancement throughout all phases of CEUS procedures.

Heterogeneous enhancement was observed in 16 lesions (51.6\%) with the features of heterogeneous perfusion of contrast agent in the early arterial phase and hyper-enhancement or iso-enhancement in the margins of the lesions. There were also small non-enhancement honeycomb-like areas among these 16 lesions. The hyperenhancement region showed partly washout in the arterial or parenchymal phase. The hypo-enhancement or non-enhancement was considered as the feature of the parenchymal phase (Figure 1B - D). The left five lesions 

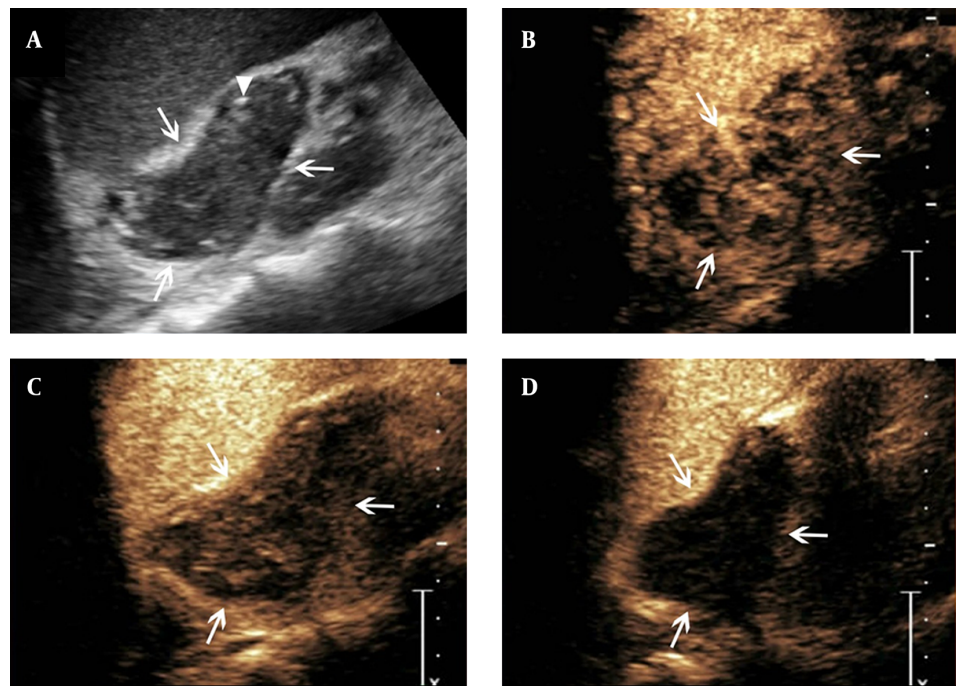

Figure 1. Features of contrast-enhanced ultrasound of adrenal tuberculosis in a lesion with heterogeneous enhancement. A, conventional sonography indicates adrenal enlargement, with abnormal shape, and heterogeneous punctate strong echo (arrowhead); B - D, Twelve seconds after SonoVue injection, the lesion shows heterogeneous enhancement and cribellate feature (B, arrows), followed by hypo-enhancement in the arterial phase when the contrast agent began to wash-out (C, arrows). Then, in the parenchymal phase, almost no enhancement is observed (D, arrows).
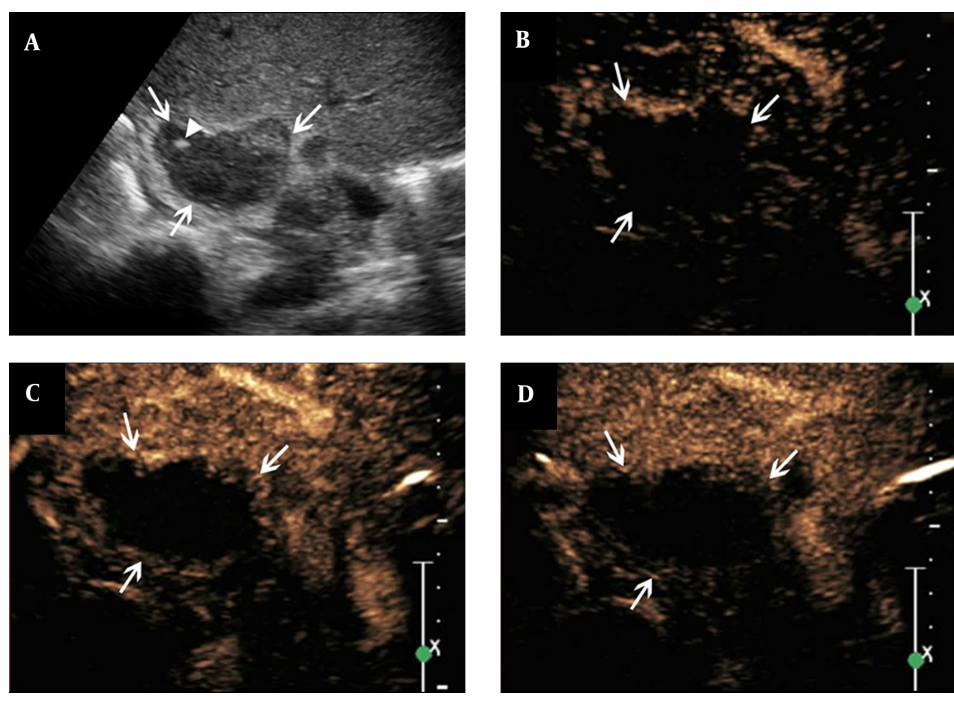

Figure 2. Features of contrast-enhanced ultrasound of adrenal tuberculosis in a lesion with non-enhancement. A, conventional sonography indicates adrenal enlargement, with abnormal shape, and heterogeneous punctate strong echo (arrowhead); B - D, The figures show that after the SonoVue injection, non-enhancement is observed throughout all phases (arrows).

showed non-enhancement throughout all phases on CEUS, which suggested no contrast agent perfusion from the early arterial to parenchymal phase (Figure $2 \mathrm{~B}-\mathrm{D}$ ). There were 10 lesions (32.3\%) showing homogeneous enhancement with the feature of homogeneous perfusion of contrast agent in the early arterial phase and homogeneous hyper-enhancement for the lesions. The enhancement showed washout in the arterial or parenchymal phase with hypo-enhancement as the main feature in the parenchymal phase for these lesions (Figure 3B - D).

In conclusion, CEUS performance of the adrenal tuberculosis was rapid enhancement in the early arterial phase, washout in the arterial phase or parenchymal phase with small non-enhancement regions presenting as honeycomb-like heterogeneous enhancement or nonenhancement in the whole regions. CEUS found detailed 

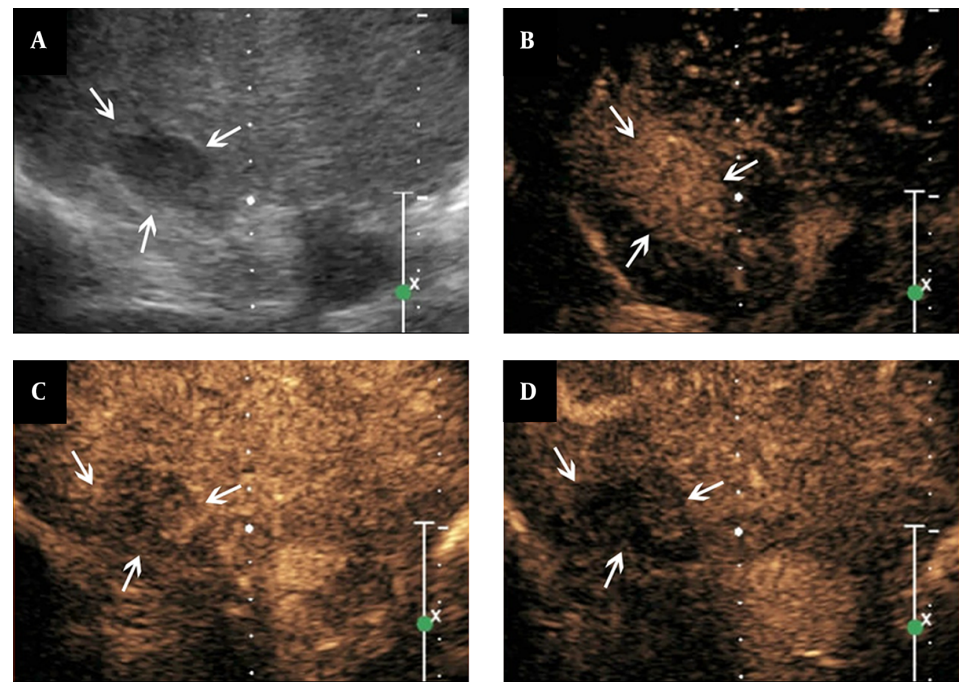

Figure 3. Features of contrast-enhanced ultrasound of adrenal tuberculosis in a lesion with homogeneous enhancement. A, conventional sonography indicates adrenal enlargement, with one hypo-echoic nodule, clear boundary, and homogeneous internal echo; B - D, The figures show that the lesion is homogeneously enhanced eleven seconds after SonoVue injection (B, arrows), followed by quick washout of the contrast agents in the arterial phase (C, arrows). Then, hypo-enhancement is observed in the parenchymal phase (D, arrows).

patterns of lesion enhancement of adrenal gland tuberculosis and had the potential to provide useful information for the diagnosis of suspected patients.

\section{Footnotes}

Authors' Contributions: Study concept and design: Gaoyi Yang, and He Ning; analysis and interpretation of data: Gaoyi Yang, and Wenzhi Zhang; drafting of the manuscript: Gaoyi Yang; critical revision of the manuscript for important intellectual content: Gaoyi Yang, Wenzhi Zhang, and He Ning; all authors read and approved the manuscript.

\section{Funding/Support: None.}

Financial Disclosure: The authors have nothing to declare.

\section{References}

1. Ozvaran MK, Baran R, Tor M, Dilek I, Demiryontar D, Arinc S, et al. Extrapulmonary tuberculosis in non-human immunodeficiency virusinfected adults in an endemic region. Ann Thorac Med.2007;2(3):118-21. doi: 10.4103/1817-1737.33700. [PubMed: 19727358].

2. Alevritis EM, Sarubbi FA, Jordan RM, Peiris AN. Infectious causes of adrenal insufficiency. South Med J. 2003;96(9):888-90. doi: 10.1097/01.SMJ.0000073269.49575.DF. [PubMed: 14513986].

3. Lam KY, Lo CY. A critical examination of adrenal tuberculosis and a 28year autopsy experience of active tuberculosis. Clin Endocrinol (Oxf). 2001;54(5):633-9. [PubMed: 11380494].

4. Dietrich CF, Ignee A, Barreiros AP, Schreiber-Dietrich D, Sienz M, Bojunga J, et al. Contrast-enhanced ultrasound for imaging of adrenal masses. Ultraschall Med. 2010;31(2):163-8. doi: 10.1055/s-0028-1109357. [PubMed: 19401979].

5. Friedrich-Rust M, Schneider G, Bohle RM, Herrmann E, Sarrazin C, Zeuzem S, et al. Contrast-enhanced sonography of adrenal masses: differentiation of adenomas and nonadenomatous lesions. AJR Am J Roentgenol. 2008;191(6):1852-60. doi: 10.2214/AJR.07.3565. [PubMed: 19020259].

6. Slonina J, Nienartowicz E, Agrawal AK, Malczewska J, Moron K. [The usefulness of contrast-enhanced sonography in the differential diagnostic of adrenal tumors]. Endokrynol Pol.2006;57(3):230-6. [PubMed: 16832787]. 